\title{
Aberrant functional connectivity of default-mode network in type 2 diabetes patients
}

\author{
Ying Cui $^{1} \cdot$ Yun Jiao $^{1} \cdot$ Hua-Jun Chen ${ }^{1} \cdot$ Jie Ding $^{1} \cdot$ Bing Luo $^{1} \cdot$ Cheng-Yu Peng ${ }^{1}$. \\ Sheng-Hong Ju ${ }^{1}$ Gao-Jun Teng ${ }^{1}$
}

Received: 14 December 2014 /Revised: 8 March 2015 / Accepted: 26 March 2015 /Published online: 23 April 2015

(C) The Author(s) 2015. This article is published with open access at Springerlink.com

\begin{abstract}
Objectives Type 2 diabetes mellitus is associated with increased risk for dementia. Patients with impaired cognition often show default-mode network disruption. We aimed to investigate the integrity of a default-mode network in diabetic patients by using independent component analysis, and to explore the relationship between network abnormalities, neurocognitive performance and diabetic variables.

Methods Forty-two patients with type 2 diabetes and 42 wellmatched healthy controls were included and underwent resting-state functional MRI in a 3 Tesla unit. Independent component analysis was adopted to extract the default-mode network, including its anterior and posterior components. Zmaps of both sub-networks were compared between the two groups and correlated with each clinical variable.

Results Patients showed increased connectivity around the medial prefrontal cortex in the anterior sub-network, but decreased connectivity around the posterior cingulate cortex in the posterior sub-network. The decreased connectivity in the posterior part was significantly correlated with the score on Complex Figure Test-delay recall test $(r=0.359, p=0.020)$, the time spent on Trail-Making Test-part B $(r=-0.346, p=$ $0.025)$ and the insulin resistance level $(r=-0.404, p=0.024)$.
\end{abstract}

Electronic supplementary material The online version of this article (doi:10.1007/s00330-015-3746-8) contains supplementary material, which is available to authorized users.

Gao-Jun Teng

gjteng@vip.sina.com

Jiangsu Key Laboratory of Molecular and Functional Imaging, Department of Radiology, Zhongda Hospital, Medical School of Southeast University, 87 Dingjiaqiao Road,

Nanjing 210009, Jiangsu, China
Conclusion Dissociation pattern in the default-mode network was found in diabetic patients, which might provide powerful new insights into the neural mechanisms that underlie the diabetes-related cognitive decline.

Key points

- Type 2 diabetes mellitus is associated with impaired cognition

- Default- mode network plays a central role in maintaining normal cognition

- Network connectivity within the default mode was disrupted in type 2 diabetes patients

- Decreased network connectivity was correlated with cognitive performance and insulin resistance level

- Disrupted default-mode network might explain the impaired cognition in diabetic population

Keywords Type 2 diabetes mellitus $\cdot$ Cognition $\cdot$ Default mode network $\cdot$ Insulin resistance $\cdot$ Resting-state functional MRI

Abbreviations
$\begin{array}{ll}\text { T2DM } & \text { Type 2 diabetes } \\ \text { FMRI } & \text { Functional magnetic resonance imaging } \\ \text { BOLD } & \text { Blood oxygenation level-dependent } \\ \text { ICN } & \text { Intrinsic connectivity network } \\ \text { DMN } & \text { Default-mode network } \\ \text { aDMN } & \text { Anterior DMN } \\ \text { pDMN } & \text { Posterior DMN } \\ \text { FC } & \text { Functional connectivity } \\ \text { MPFC } & \text { Medial pre-frontal cortex } \\ \text { ACC } & \text { Anterior cingulate cortex } \\ \text { PCC } & \text { Posterior cingulate cortex } \\ \text { AD } & \text { Alzheimer's disease } \\ \text { ICA } & \text { Independent component analysis }\end{array}$




$\begin{array}{ll}\text { MMSE } & \text { Mini-Mental State Examination } \\ \text { HAM-D } & \text { Hamilton Depression Rating Scale } \\ \text { OGTT } & \text { Oral glucose tolerance test } \\ \text { IR } & \text { Insulin Resistance } \\ \text { AVLT } & \text { Auditory Verbal Learning Test } \\ \text { CFT } & \text { Complex Figure Test } \\ \text { VFT } & \text { Verbal Fluency Test } \\ \text { TMT } & \text { Trail Making Test } \\ \text { CDT } & \text { Clock Drawing Test } \\ \text { FLAIR } & \text { Fluid-attenuated inversion recovery } \\ \text { WMH } & \text { White matter hyperintensity } \\ \text { MNI } & \text { Montreal Neurological Institute } \\ \text { IC } & \text { Independent component } \\ \text { ROI } & \text { Region of interest } \\ \text { FDR } & \text { False discovery rate } \\ \text { IPL } & \text { Inferior parietal lobule } \\ \text { SFG } & \text { Superior frontal gyrus }\end{array}$

\section{Introduction}

Type 2 diabetes mellitus (T2DM) is associated with accelerated cognitive decline [1] and is a risk factor for dementia [2]. Recently, an increasing number of studies have provided significant insights into the structural and functional changes in the diabetic brain [3-5], which suggests the potential for neuroimaging to assist in understanding the pathways that might link T2DM to cognitive decrements.

Resting-state functional magnetic resonance imaging (rsfMRI) has enabled the evaluation of brain functional architecture on the basis of blood oxygenation level-dependent (BOLD) signal, which represents the spontaneous neuronal activity at baseline state [6]. Resting-state networks, namely intrinsic connectivity networks (ICNs), are defined as brain regions with highly correlated time courses of low-frequency $(<0.1 \mathrm{~Hz})$ BOLD signal fluctuations [7]. Among these networks, the default-mode network (DMN) is the most widely studied and well-characterized one. Consisting of the medial pre-frontal cortex (MPFC), anterior cingulate cortex (ACC), posterior cingulate cortex (PCC) and precuneus [8], DMN is suggested as a major contributor to the normal cognitive functioning [9]. Reduction of intrinsic connectivity of the DMN has been observed in a number of mental disorders such as Alzheimer's disease (AD), autism, schizophrenia and hepatic encephalopathy $[8,10-12]$, which suggests its potential as a biomarker for clinical application. Given the impaired cognitive functioning in diabetic patients, we hypothesized that there might also be alterations in DMN connectivity in this population.

Independent component analysis (ICA), one of the multiple-regression methods, is a robust technique based on blind source separation that captures the essential components of multivariate rs-fMRI signals [13]. It helps to separate the whole-brain signal fluctuations from physiological noise and automatically capture the entire DMN as a single major component [14]. In comparisons with other analytical methods such as the seed-based method, ICA is proved to decrease the heterogeneity of DMN pattern by avoiding the a-priori seed selection, thus raising the reliability and reproducibility of the results [2].

In the present study, we adopted the ICA method to automatically identify DMN and explore its connectivity in T2DM patients. We aimed to evaluate (1) whether T2DM is associated with disrupted DMN compared with the healthy controls; and (2) whether the functional connectivity (FC) changes in the DMN are correlated with worse neurocognitive performance and T2DM-related parameters.

\section{Methods}

\section{Participants}

This study was approved by the local ethics committee and conducted between November 2012 and November 2013. Written informed consent was obtained from all participants. All subjects were between 50 and 75 years of age, righthanded and without any MR contraindication. A total of 43 patients were recruited by advertisements and met the latest criteria of T2DM [15]. All patients had a diabetes duration of at least 1 year. They were in close self-monitoring and routinely treated with various hypoglycaemic agents; none had any history of hypoglycaemic episodes. Healthy controls ( $\mathrm{n}=$ 42) were also recruited through advertisements and were matched with T2DM patients with respect to age, sex and education. Exclusion criteria for both groups included an indication of dementia based on a Mini-Mental State Examination (MMSE) score $\leq 24$ [16], history of depression based on the Hamilton Depression Rating Scale (HAM-D) score $>7$ [17], other psychiatric or neurological disorder unrelated to diabetes, a past history of alcohol or substance abuse, and brain lesions such as tumour or stroke. According to previous studies [18], we intentionally did not exclude subjects with vascular risk factors to raise the generalizability of our results.

Biometric measurement was performed at 8:00 a.m. All participants were recorded with detailed history of hypertension, smoking, medication and vascular disease. Laboratory parameters, such as fasting plasma glucose (FPG), fasting $\mathrm{HbA1c}$, insulin and cholesterol were obtained and measured with standard laboratory testing. Subsequently, an oral glucose tolerance (OGTT) test (75 g dextrose monohydrate in $250 \mathrm{~mL}$ water) was performed on all healthy controls and patients without receiving treatment $(n=8)$ to verify the diagnosis and severity of T2DM. Control subjects with fasting blood glucose level $\geq 7.0 \mathrm{mmol} / \mathrm{L}$ or 2 -h postprandial glucose level $\geq 7.8 \mathrm{mmol} / \mathrm{L}$ after OGTT test were excluded. During the 
interview, blood pressure was measured at three different time points and averaged. Hypertension was defined as previously described [19]. HOMA-IR was used to determine the level of insulin resistance (IR) for all subjects, except for those with insulin treatment. Diabetic complications including the retinopathy and peripheral neuropathy were also measured to get a full picture of glycemic control in T2DM patients, using the same methods and criteria described elsewhere [20,21].

\section{Neuropsychological assessment}

All participants performed an extensive neurocognitive examination covering several cognitive domains. All tests took approximately $60 \mathrm{~min}$ to complete. No medication was allowed immediately before the cognitive assessment. The MMSE and the HAM-D were used for excluding possible dementia and depression, respectively $[16,17]$. The Auditory Verbal Learning Test (AVLT) and Rey-Osterrieth Complex Figure Test (CFT) (both included immediate recall and delayed recall tasks) were used for assessing episodic memory regarding either verbal or visual information [22, 23]. The Verbal Fluency Test (VFT) examines the semantic memory [24]. The Trail Making Test, parts A and B (TMT-A and TMT-B), was primarily used to evaluate attention and psychomotor speed [25]. The Clock Drawing Test (CDT) draws on a number of cognitive domains such as executive function and working memory [26].

\section{Image acquisition and assessment}

MRI data were acquired using a Siemens Verio 3.0-T system (Siemens, Erlangen, Germany) with a 12-channel head coil. All subjects had to refrain from taking medication before the MR. During imaging, subjects were covered with eye masks, instructed to remain awake and keep their heads still. Soft pads were used to reduce head movement and headphones were used to reduce noise. Functional images were obtained interleaved by using a gradient-recalled echo-planar (GREEPI) sequence (repetition time $2000 \mathrm{~ms}$, echo time $25 \mathrm{~ms}$, flip angle of $90^{\circ}$, field of view $240 \mathrm{~mm} \times 240 \mathrm{~mm}$, acquisition matrix $=64 \times 64)$. A total of 240 whole-brain volumes were collected on 36 oblique slices with 4-mm thickness. Structural images were obtained by using a high-resolution T1-weighted 3D magnetization-prepared rapid gradient-echo imaging (3D MPRAGE) (176 slices, repetition time $1900 \mathrm{~ms}$, echo time $2.48 \mathrm{~ms}$, slice thickness $1.0 \mathrm{~mm}$, flip angle of $9^{\circ}$, inversion time $900 \mathrm{~ms}$, field of view $250 \times 250 \mathrm{~mm}$, in-plane resolution $256 \times 256$ ) for image registration and functional localization. Fluid-attenuated inversion recovery (FLAIR) images were obtained with the following parameters: repetition time $8500 \mathrm{~ms}$, echo time $94 \mathrm{~ms}, 20$ slices with 5 -mm thickness, voxel size $1.3 \times 0.9 \times 5 \mathrm{~mm}^{3}$.
The white matter hyperintensity (WMH) on the FLAIR images was assessed using the age-related white matter changes scale [27]. Participants with a rating score of above one (confluence of lesions or diffuse involvement of the entire region) were excluded. Lacunar infarct was also assessed as well-defined areas of $>2 \mathrm{~mm}$ with signal of cerebrospinal fluid.

\section{Functional image processing and identification of DMNs}

FMRI data preprocessing was carried out using SPM8 (Statistical Parametric Mapping, http://www.fil.ion.ucl.ac.uk/ $\mathrm{spm} /$ ). The following procedures were applied: slice timing adjustment; realignment for head motion correction (subjects with head motion $>2.0-\mathrm{mm}$ translation or $>2.0^{\circ}$ rotation were excluded); spatial normalization to standard Montreal Neurological Institute (MNI) template with a resampling voxel size of $3 \times 3 \times 3 \mathrm{~mm}^{3}$; spatial smoothing with a Gaussian kernel of $4 \mathrm{~mm}$.

ICA was conducted using the Informix algorithm in the Group ICA of fMRI Toolbox (GIFT) software (Medical Image Analysis Lab, University of New Mexico, Albuquerque, NM, USA; http://icatb.sourceforge.net/). The number of independent components (ICs) was determined by using the minimum description length criteria and the mean dimension estimation was 25 . Then, the averaged IC number was used for each subject for ICA decomposition. For each IC, the time courses correspond to the waveform of a specific pattern of coherent brain activity, and the intensity of this pattern of brain activity across the voxels is expressed in the associated spatial map. To display voxels relevant to each particular IC, the intensity values in each map were converted to $\mathrm{z}$ values. As previously described [12], the final DMN component was identified by visual inspection on the basis of periodic temporal fluctuation, spatial pattern, and distinct peak of power spectrum at low-frequency $(<0.1 \mathrm{~Hz})$ range.

\section{Statistical analysis}

The differences of clinical variables and cognitive performance between patients and the control group were examined by independent two-sample $t$ test for continuous variables and $\chi 2$ test for proportions. Each comparison was performed with the SPSS software (version 18.0; SPSS, Inc., Chicago, IL, USA). $P<0.05$ was considered to be statistically significant.

To assess the within-group integrity of the best-fit components, one-sample $t$-tests were performed on the spatial maps of DMN for each group by using REST-Statistical Analysis (written by Chaogan Yan, www.restfmri.net) [28]. $P$ values less than 0.05 were considered statistically significant. Multiple comparisons were performed by the relatively conservative false discovery rate (FDR) criterion to acquire the most significant results that mainly contain the DMN regions. 
To explore the between-group differences, two-sample $t$ tests were performed on the spatial maps of the DMN in a voxel-by-voxel manner. To restrict the comparison to the voxels within the DMN only, we applied a mask that was created by combining the results obtained from one-sample $t$-test in each group. To exclude the possible confounding effects, age, sex and education level were included as covariates. Statistical threshold was set with $P<0.05$, combined with a minimum cluster size of 49 voxels for the anterior DMN, and 43 voxels for the posterior DMN for multiple comparison correction, resulted in a corrected $P<0.05$. To reflect the intrinsic abnormality of T2DM as much as possible, a less conservative correction was chosen that is determined by Monte Carlo simulation (AlphaSim program in AFNI, http://afni.nih. gov/afni/docpdf/AlphaSim.pdf) with the following parameters: single voxel, $P=0.05$; full width at half maximum, $4 \mathrm{~mm}$; with the one-sample $t$-test results as the mask.

To investigate the relationship between DMN connectivity and clinical variables, Pearson correlation analyses were performed in a region of interest (ROI)-wise manner. Specifically, the ROI was defined as a spherical region within $6 \mathrm{~mm}$ of the peak activation in each brain region that differed significantly between the two groups. Then the mean $\mathrm{z}$ values of each ROI were correlated against the cognitive performance and T2DMrelated variables in each patient. All analyses were performed using the SPSS software. $P$ values $<0.05$ were considered statistically significant. To reduce the chance of type I error, a Bonferroni correction was performed for multiple comparisons.

To explore the effects of treatment modality in patients group, DMN pattern was first compared between patients with and without insulin therapy [29]. Next, we also explored the effects of diabetic complications by comparing the DMN pattern among the patients with neuropathy, retinopathy and those without complications. Finally, to determine whether these intergroup varieties could explain the DMN differences between T2DM patients and healthy controls, FC values were extracted from brain regions that differed significantly between groups and compared among all these sub-groups of patients.

\section{Results}

\section{Participant information}

One T2DM patient was excluded because of excessive head movement. Therefore, a total of 84 participants (42 T2DM patients and 42 healthy controls) were included in the final data analysis. Demographic and cognitive characteristics of all participants are presented in Table 1. Among the patients, 11 were treated with insulin, while the remainder were treated with either oral hypoglycaemic agents $(n=23)$ or dietary restriction only $(n=8)$. Clinical variables did not differ significantly between patients with and without insulin treatment, but the former tended to perform worse on several cognitive tests (supplementary Table 1). Comparing the diabetic and control groups, no significant differences were observed in terms of the total cholesterol, WMH loads and lacunar infarcts, and body mass index (BMI). However, patients performed significantly worse on the TMT-B and CFT-delayed recall tests, which mainly involved cognitive domains of executive function, processing speed and spatial episodic memory. Correlation analyses indicated that disease duration and HOMA-IR, instead of hyperglycaemic indices themselves (FPG and $\mathrm{HbAlc}$ ), were the main contributors to these impaired performance (Supplementary Table 2).

\section{Spatial patterns of DMN}

Among the 25 components estimated during the data processing, two components were identified as the two sub-networks of DMN. A one-sample $t$-test showed the typical spatial pattern of each sub-network in both groups $(P<0.05$, FDR corrected) (Fig. 1). In the control group, the anterior DMN (Fig. 1, lower row) displayed functional connectivity (FC) mainly in the MPFC areas, as well as in the PCC/precuneus and small inferior parietal lobules (IPL) clusters. The posterior DMN (Fig. 1, lower row) showed FC within the PCC, precuneus and bilateral IPL regions, but also included a small part of MPFC. The DMN pattern of the patients largely included the same brain regions as the healthy controls.

\section{Group differences in DMN}

Compared with the healthy controls, patients showed increased $\mathrm{FC}$ in the anterior $\mathrm{DMN}$ region and decreased $\mathrm{FC}$ in the posterior DMN region $(P<0.05$, AlphaSim corrected). Specifically, the increased connectivity mainly involved bilateral superior frontal gyrus (SFG) (Fig. 2A). The decreased connectivity included the PCC and the precuneus (Fig. 2B). A detailed list of brain regions with aberrant FC in each subnetwork is presented in Table 2.

DMN connectivity map was further compared between patients under insulin and non-insulin treatment, and between those with different diabetic complications. No significant difference was observed among these sub-groups of patients. Similarly, FC values extracted from the frontal region and the posterior regions also showed no significant difference among these sub-groups (Fig. 3A and B). However, insulintreated patients suffered more functional alterations than those under non-insulin therapy, as shown in Fig. 3A. 
Table 1 Demographic, clinical and cognitive characteristics

\begin{tabular}{|c|c|c|c|}
\hline Characteristics & $\begin{array}{l}\text { T2DM Patients } \\
(\mathrm{n}=42)\end{array}$ & $\begin{array}{l}\text { Control subjects } \\
(\mathrm{n}=42)\end{array}$ & $P$ value \\
\hline Age (years) & $60.4 \pm 7.0$ & $58.2 \pm 6.3$ & 0.14 \\
\hline Sex (male) & $23(55)$ & $14(33)$ & 0.08 \\
\hline Education (years) & $9.6 \pm 3.8$ & $10.2 \pm 2.3$ & 0.35 \\
\hline Disease duration (years) & $9.4 \pm 4.7$ & - & \\
\hline Fasting glucose $(\mathrm{mmol} / \mathrm{L})^{\mathrm{a}}$ & $7.8 \pm 2.1$ & $5.5 \pm 0.4$ & $<0.01$ \\
\hline 2-h postprandial glucose $(\mathrm{mmol} / \mathrm{L})^{\mathrm{a}}$ & $15.5 \pm 4.2(\mathrm{n}=8)$ & $6.5 \pm 1.7$ & $<0.01$ \\
\hline $\mathrm{HbA1c}(\%)(\mathrm{mmol} / \mathrm{mol})^{\mathrm{a}}$ & $7.9 \pm 1.6(62.7 \pm 17.4)$ & $5.6 \pm 0.3(37.8 \pm 3.6)$ & $<0.01$ \\
\hline HOMA-IR ${ }^{\mathrm{a}}$ & $3.4 \pm 2.0$ & $2.5 \pm 1.3$ & 0.03 \\
\hline \multicolumn{4}{|l|}{ Blood pressure } \\
\hline Systolic (mmHg) & $136.6 \pm 14.7$ & $132.7 \pm 15.0$ & 0.23 \\
\hline Diastolic (mmHg) & $85.8 \pm 11.3$ & $86.8 \pm 11.4$ & 0.70 \\
\hline Triglycerides $(\mathrm{mmol} / \mathrm{L})$ & $1.46 \pm 0.80$ & $1.41 \pm 0.87$ & 0.77 \\
\hline Total cholesterol $(\mathrm{mmol} / \mathrm{L})$ & $5.3 \pm 1.1$ & $5.2 \pm 0.9$ & 0.69 \\
\hline $\operatorname{BMI}\left(\mathrm{kg} / \mathrm{m}^{2}\right)$ & $24.7 \pm 2.9$ & $24.0 \pm 2.8$ & 0.29 \\
\hline White matter lesions & $1(0-6)$ & $0(0-7)$ & 0.28 \\
\hline Lacunar infarcts & $9(21)$ & $5(12)$ & 0.38 \\
\hline \multicolumn{4}{|l|}{ Cognitive performance } \\
\hline MMSE & $28.3 \pm 1.1$ & $28.7 \pm 1.2$ & 0.11 \\
\hline Trail Making Test-A(s) & $67.8 \pm 22.3$ & $62.2 \pm 15.1$ & 0.16 \\
\hline Trail Making Test-B(s) ${ }^{\mathrm{a}}$ & $178.1 \pm 56.0$ & $149.3 \pm 51.7$ & 0.01 \\
\hline Auditory Verbal Memory Test & $5.9 \pm 1.4$ & $6.5 \pm 2.0$ & 0.08 \\
\hline $\begin{array}{l}\text { Auditory Verbal Memory Test-Delay } \\
\text { Recall }\end{array}$ & $5.7 \pm 2.5$ & $6.3 \pm 2.2$ & 0.20 \\
\hline Clock Drawing Test & $3.3 \pm 0.6$ & $3.5 \pm 0.5$ & 0.10 \\
\hline Complex Figure Test-Immediate Recall & $34.3 \pm 1.5$ & $34.9 \pm 1.4$ & 0.07 \\
\hline Complex Figure Test-Delayed Recall ${ }^{\mathrm{a}}$ & $13.3 \pm 5.9$ & $17.7 \pm 6.0$ & $<0.01$ \\
\hline Verbal Fluency Test & $16.8 \pm 3.8$ & $17.5 \pm 3.1$ & 0.55 \\
\hline Hamilton Depression Rating Scale & $1.7 \pm 1.0$ & $1.2 \pm 0.8$ & 0.20 \\
\hline
\end{tabular}

Data are represented as mean $\pm \mathrm{SD}, \mathrm{n}(\%)$ or median (range). T2DM, type 2 diabetes mellitus

${ }^{\text {a }} P$ value $<0.05$

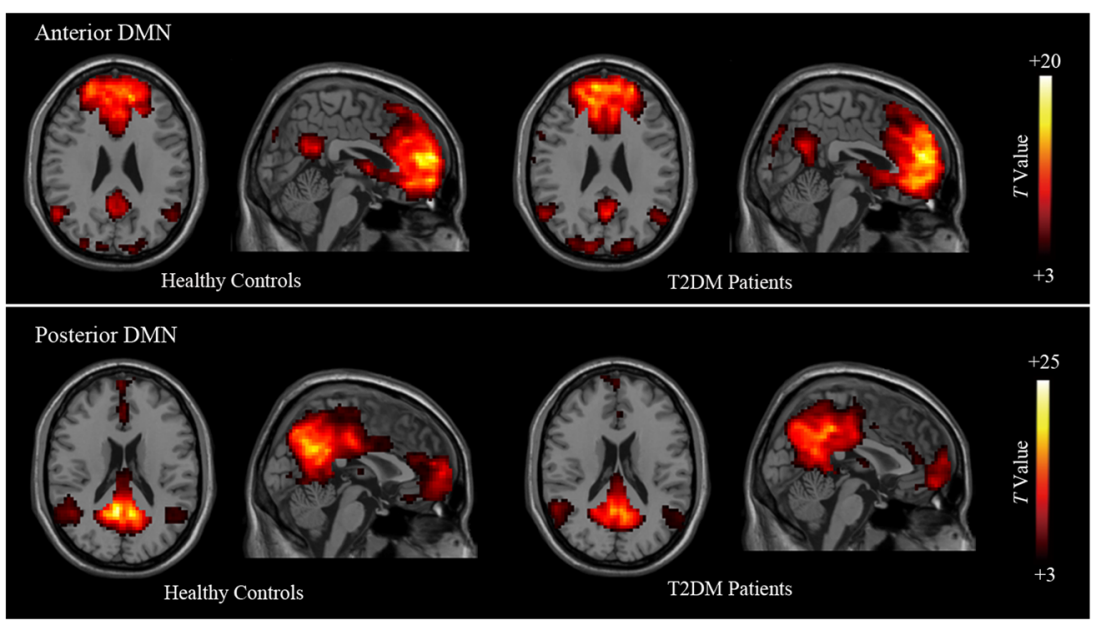

Fig. 1 Results of one-sample t-test of the two components representing the default-mode sub-networks in patients and healthy groups $(P<0.05$, FDR corrected). Representative images of the anterior default-mode network (DMN) (upper row) and the posterior DMN (lower row) in healthy controls and type 2 diabetes mellitus (T2DM) patients. Both components are displayed from the sectional and sagittal views. Colour scale represents $t$-values in each component $(P<0.05$, FDR corrected). DMN, default-mode network; T2DM, type 2 diabetes 


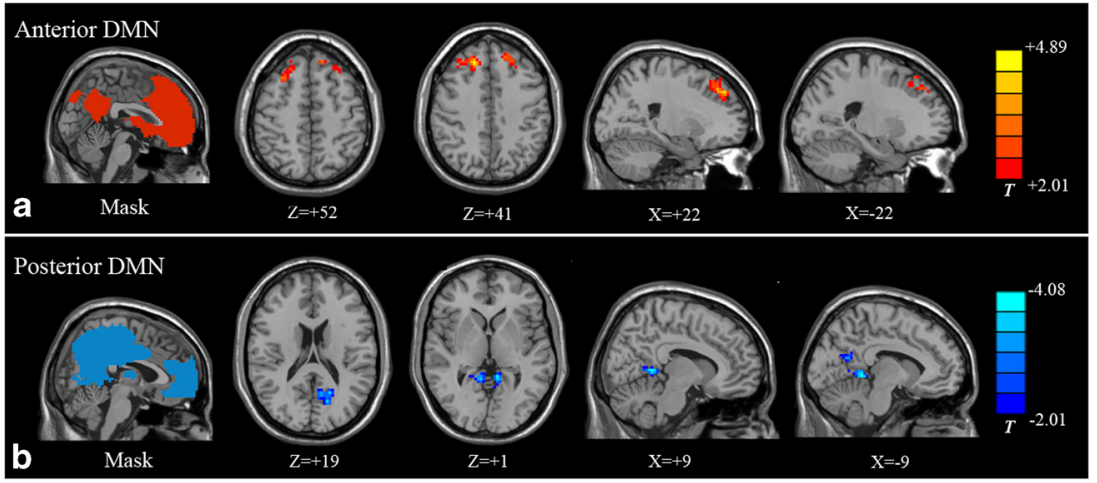

Fig. 2 Comparison of default-mode network (DMN) sub-networks between type 2 diabetes mellitus (T2DM) patients and healthy controls. (A) Compared with the healthy controls, patients with T2DM showed significantly increased FC (red colour) within the anterior DMN sub-network, predominantly in the right and the left SFG. (B) Decreased FC (blue colour) was found in the posterior sub-network of the DMN, which was mainly located around the retrosplenial regions such as the PCC and the precuneus. Pictures on the left column are masks of the two subcomponents of DMN, obtained by combining results of one-sample $t$-test in each group, thresholding at $P<0.05$ (FDR corrected). Colour scale represents $t$-values. FC, functional connectivity; SFG, superior frontal gyrus; PCC, posterior cingulate cortex

\section{Correlation analysis}

FC strength in the pDMN was positively correlated with the CFT-delay and TMT-B performance, and negatively correlated with the insulin resistance level (Fig. 4). Specifically, the hypoconnectivity in the PCC region was related to higher insulin resistance level $(\mathrm{r}=-0.404, \mathrm{p}=0.024)$ (Fig. 4B), while the mean $\mathrm{z}$ value in the precuneus had positive correlations with the CFT-delay score $(r=0.395, p=0.020)$ and negative correlations with the time spent on TMT-B $(\mathrm{r}=-0.346, \mathrm{p}=$ 0.025) (Fig. 4C and D). No such correlations were observed in the control group (Supplementary Table 2). The treatment modality (with and without insulin) did not alter the significance of the associations between clinical variables and imaging parameters (Supplementary Table 3).

Table 2 Brain regions with significant differences on default-mode network (DMN) connectivity between type 2 diabetes mellitus (T2DM) patients and healthy controls

\begin{tabular}{lrrrrrrr}
\hline Brain region & BA & \multicolumn{2}{l}{ MNI coordinates } & \multirow{2}{*}{$\begin{array}{l}\text { Cluster } \\
\text { size }\end{array}$} & $T$ value \\
\cline { 3 - 5 } & & X & Y & Z & & \\
\hline Anterior DMN & & & & & & \\
$\quad$ R superior frontal gyrus & 9 & 18 & 33 & 42 & 188 & +4.89 \\
$\quad$ L superior frontal gyrus & 8 & -12 & 39 & 48 & 73 & +3.15 \\
Posterior DMN & & & & & & \\
$\quad \begin{array}{l}\text { Precuneus } \\
\quad \text { Posterior cingulate cortex }\end{array}$ & 31 & -15 & -69 & 18 & 84 & -3.40 \\
& & 9 & -48 & 3 & 82 & -4.08
\end{tabular}

BA, Brodmann area; MNI, Montreal Neurological Institute; X, Y, Z, coordinates of primary peak locations in the MNI space; $\mathrm{L}$ indicates the left; $\mathrm{R}$ indicates the right; positive $t$-values: T2DM $>$ control subjects; negative $t$-values: T2DM $<$ control subjects

Comparisons were performed at $P<0.05$, corrected for multiple comparisons

\section{Discussion}

Using the ICA method, we demonstrated a disrupted pattern of resting-state DMN in T2DM patients. Compared with healthy controls, T2DM patients showed increased FC within the aDMN regions while decreased $\mathrm{FC}$ within the $\mathrm{pDMN}$ regions. These functional alterations were related to the impaired cognitive performance and the increased insulin resistance level.

Evidence from recent fMRI studies suggested that the DMN anterior and posterior sub-networks interact in a dynamic equilibrium, which is important in the maintenance of normal cognition functioning [30]. However, with advancing age, this equilibrium is disrupted and these sub-networks develop greater independence from each other, thus resulting in their reciprocal changes [31]. Similar changes were also observed in other mental disorders, including Alzheimer's disease, ApoE 4 allele carriers, traumatic brain injury, major depression, epilepsy and autism [12,31-36], suggesting that multiple disorders are linked to some analogous disruption of the DMN. Our study, for the first time, suggested this dissociation pattern in T2DM patients. Given its consistency across different mental disorders, this DMN dissociation pattern might be a potential imaging biomarker to identify T2DM patients with cognitive impairment. Furthermore, recent neuroimaging studies revealed that DMN changes could predict treatment response in mental disorders such as schizophrenia [37]. Therefore, this disrupted DMN signature also holds great potential to provide prognostic information for T2DMassociated cognitive decline.

Decreased connectivity was predominantly located in the PCC and the precuneus. These regions are the central nodes of the brain, the impairments of which are frequently treated as the early signs of cognitive impairment [38]. Neural dysfunction in these regions has been reported in the diabetic 


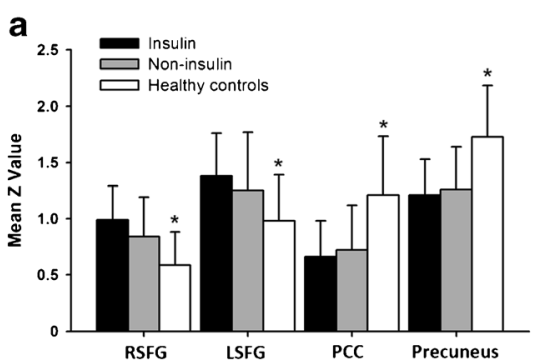

Fig. 3 Default-mode network (DMN) connectivity differences among different patients sub-groups and healthy controls. (A). The mean FC values extracted from the frontal regions in the diabetic group were significantly higher than the control group, while the mean FC values of the occipital lobe were significantly lower. There is a trend that patients with insulin treatment suffered more functional alterations than those without,

population. For example, decreased neural intensity and coherence were detected around the precuneus regions [39]; decreased connectivity between the PCC and other nodes within the DMN was also observed [40]. Together with these previous findings, our results provided further evidence for the neural dysfunction in diabetes. Being correlated with cognitive performance, hypoconnectivity in these posterior regions might provide important insights into the neural mechanism underlying the impaired cognition in diabetic population. On the other hand, significantly increased connectivity was found in the bilateral superior frontal gyrus (SFG). Increased SFG activation has been reported previously in several depressionrelated fMRI studies, given that it is responsible for the modulation of emotional behavior and self-referential processing

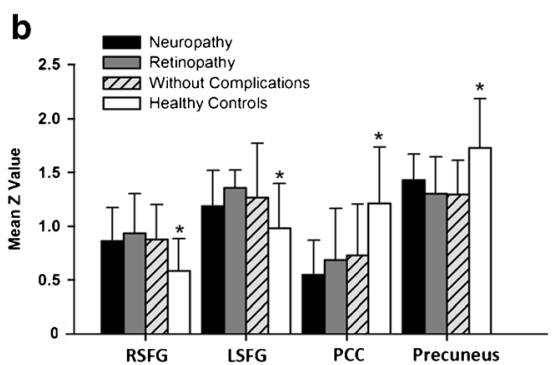

although the differences were not significant. (B) The mean FC value extracted from the frontal and the occipital lobe did not differ between the patients with and without diabetic complications. FC, functional connectivity; RSFG, right superior frontal gyrus; LSFG, left superior frontal gyrus; PCC, posterior cingulate cortex

[41]. T2DM is known to be associated with an increased risk of depression, which could lead to impaired self-consciousness and depressive symptoms [42]. We thus assume that the increased MPFC connectivity might be an early change that occurs before clinically measurable depression. Further studies including depressive T2DM patients are of particular interest to determine the clinical significances of our results.

Our results showed no correlations between the glycaemic control and functional changes, which was inconsistent with a previous PET/CT study [43]. It is important to note that whether hyperglycaemia is an independent risk factor for impaired cognition is still under debate [44]. As 'diabetic encephalopathy' is a multifactorial condition [45], the hyperglycaemia per se may not be the most important
Fig. 4 Correlations between clinical parameters and the mean $\mathrm{z}$ value extracted from significantly different brain regions. (A) Spherical regions of interest (ROIs) located in the precuneus and the PCC, and their coordinates of peak activation.

(B) The mean $\mathrm{z}$ value of the ROI extracted from the PCC was negatively related to insulin resistance $(\mathrm{R}=0.404, \mathrm{P}=0.024)$.

(C) The mean $\mathrm{z}$ value of the ROI extracted from the precuneus was positively correlated with the CFT-delayed recall scores in the diabetic group $(R=0.395$, $P=0.020$ ). (D) The mean $\mathrm{z}$ value of the ROI extracted from the precuneus had negative correlations with the time spent on the TMT-B $(R=-0.346$, $P=0.025)$. CFT, complex figure test; TMT-B, trail making testpart B; PCC, posterior cingulate cortex a

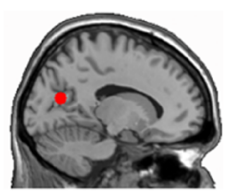

Precuenus $(x=-15, y=-69, z=48)$

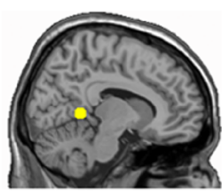

$\mathrm{PCC}$ $(\mathrm{x}=9, \mathrm{y}=-48, \mathrm{z}=3)$ b

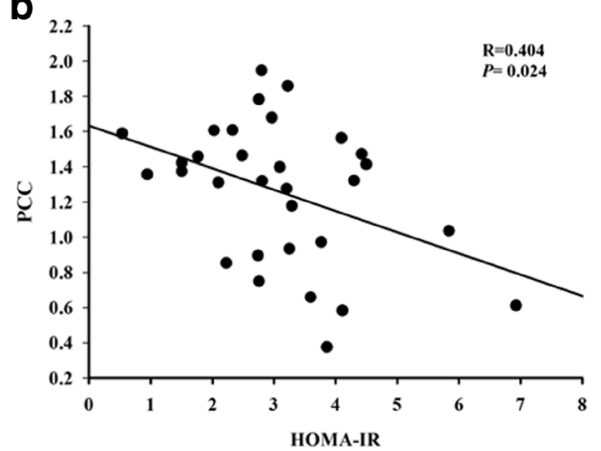

C

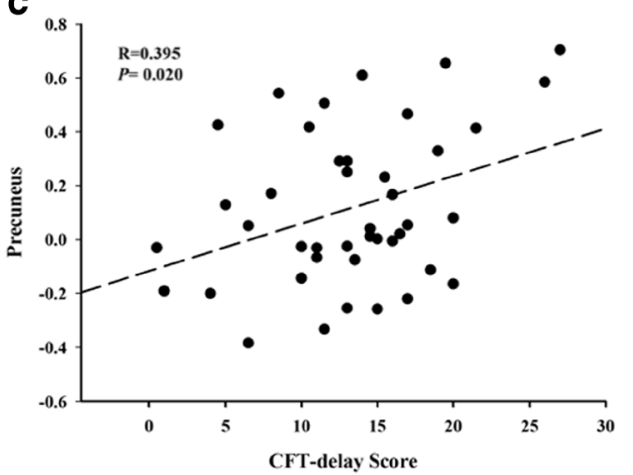

d

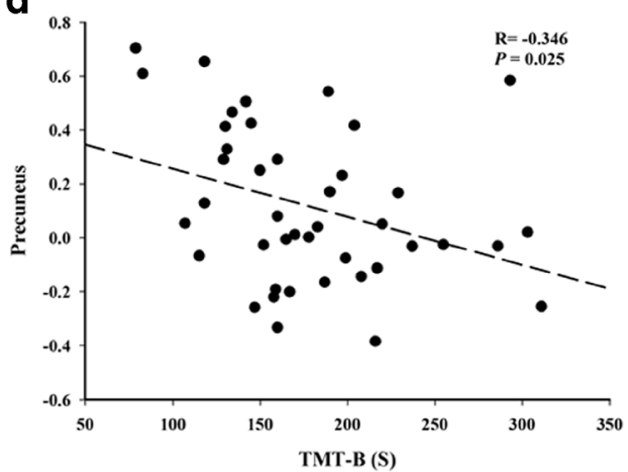


contributor for the cognitive impairment. In the current results, IR was independently correlated to DMN alterations (Fig. 4B). Recently, IR has been recognized as a promising determinant for cognitive decline, as it is tightly associated with Alzheimer's disease-like pathology [1]. Consistent with previous imaging studies [46, 47], our results suggested that IR might be more sensitive to detect neural alterations over other glycaemic indices. In addition, patients having insulin treatment suffered more functional abnormalities than those without (Fig. 3A). This is in accordance with previous largescale clinical studies [48] supporting that insulin-treated patients have a higher risk for dementia, which is possibly mediated by more severe pathological changes and direct effects of insulin. A critical direction for future work is to explore the mechanism underlying T2DM associated cognitive decline. Further research with longitudinal design and less heterogeneity are warranted to achieve this goal.

We note that our study has several limitations. First, this study was limited to a relatively small sample size by using advertisement recruitment, which may to some extent reduce the generalizability of the results. Second, most of the included patients were variously medicated, making it difficult to determine whether some of our results were secondary to possible effects of medication. Medication-naïve subjects should be included in future studies to exclude such effects. Finally, we did not measure the serum glucose level just before the MR. Further studies should take it into consideration to better elucidate the relationship between glycaemic control and neural dysfunction.

In conclusion, the DMN connectivity was altered in T2DM patients, which was associated with impaired cognition and increased insulin resistance. In contrast to standard cognitive tests, using DMN as a potential screening tool for cognitive decline offers us an opportunity to observe the living brain in a non-invasive way. Future perspectives include application of fMRI techniques in longitudinal studies to define the evolution of DMN connectivity with the progression of T2DM, as well as the effects of various therapeutic approaches on central nervous system plasticity.

Acknowledgements The scientific guarantor of this publication is GaoJun Teng. The authors of this manuscript declare no relationships with any companies whose products or services may be related to the subject matter of the article. This study received funding from the Major State Basic Research Development Program of China (973 Program) (Nos. 2013CB733800, 2013CB733803), Jiangsu Provincial Special Program of Medical Science (BL2013029) and National Natural Science Foundation of China General Projects (Nos. 81230034, 81271739). No complex statistical methods were necessary for this paper. Institutional Review Board approval was obtained. Written informed consent was obtained from all subjects (patients) in this study. Some study subjects or cohorts have been previously reported in "Altered spontaneous brain activity in type 2 diabetes: a resting-state functional MRI study. Diabetes 2014, 63:749-760"

Methodology: prospective, cross sectional study / diagnostic study. Performed at one institution.
Open Access This article is distributed under the terms of the Creative Commons Attribution-NonCommercial 4.0 International License (http:// creativecommons.org/licenses/by-nc/4.0/), which permits any noncommercial use, distribution, and reproduction in any medium, provided you give appropriate credit to the original author(s) and the source, provide a link to the Creative Commons license, and indicate if changes were made.

\section{References}

1. McCrimmon RJ, Ryan CM, Frier BM (2012) Diabetes and cognitive dysfunction. Lancet 379:2291-2299

2. Musen G, Jacobson AM, Bolo NR et al (2012) Resting-state brain functional connectivity is altered in type 2 diabetes. Diabetes 61 : 2375-2379

3. Reijmer YD, Brundel M, de Bresser J, Kappelle LJ, Leemans A, Biessels GJ (2013) Microstructural white matter abnormalities and cognitive functioning in type 2 diabetes: a diffusion tensor imaging study. Diabetes Care 36:137-144

4. Marder TJ, Flores VL, Bolo NR et al (2014) Task-induced brain activity patterns in type 2 diabetes: a potential biomarker for $\operatorname{cog}$ nitive decline. Diabetes 63:3112-3119

5. Ryan JP, Fine DF, Rosano C (2014) Type 2 diabetes and cognitive impairment: contributions from neuroimaging. J Geriatr Psychiatry Neurol 27:47-55

6. Fox MD, Raichle ME (2007) Spontaneous fluctuations in brain activity observed with functional magnetic resonance imaging. Nat Rev Neurosci 8:700-711

7. Rosazza C, Minati L (2011) Resting-state brain networks: literature review and clinical applications. Neurol Sci 32:773-785

8. Buckner RL, Andrews-Hanna JR, Schacter DL (2008) The brain's default network: anatomy, function, and relevance to disease. Ann N Y Acad Sci 1124:1-38

9. Smucny J, Wylie KP, Tregellas JR (2014) Functional magnetic resonance imaging of intrinsic brain networks for translational drug discovery. Trends Pharmacol Sci 35:397-403

10. Adriaanse SM, Sanz-Arigita EJ, Binnewijzend MA et al (2014) Amyloid and its association with default network integrity in Alzheimer's disease. Hum Brain Mapp 35:779-791

11. Zhang L, Qi R, Wu S et al (2012) Brain default-mode network abnormalities in hepatic encephalopathy: a resting-state functional MRI study. Hum Brain Mapp 33:1384-1392

12. Zhou Y, Milham MP, Lui YW et al (2012) Default-mode network disruption in mild traumatic brain injury. Radiology 265:882-892

13. De Luca M, Beckmann CF, De Stefano N, Matthews PM, Smith SM (2006) fMRI resting state networks define distinct modes of long-distance interactions in the human brain. NeuroImage 29: $1359-1367$

14. Greicius MD, Krasnow B, Reiss AL, Menon V (2003) Functional connectivity in the resting brain: a network analysis of the default mode hypothesis. Proc Natl Acad Sci U S A 100:253-258

15. American Diabetes Association (2013) Diagnosis and classification of diabetes mellitus. Diabetes Care 36:S67-S74

16. Galea M, Woodward M (2005) Mini-Mental State Examination (MMSE). Aust J Physiother 51:198

17. Hamilton M (1960) A rating scale for depression. J Neurol Neurosurg Psychiatry 23:56-62

18. Manschot SM, Brands AM, van der Grond J et al (2006) Brain magnetic resonance imaging correlates of impaired cognition in patients with type 2 diabetes. Diabetes 55: 1106-1113

19. van den Berg E, Kloppenborg RP, Kessels RPC, Kappelle LJ, Biessels GJ (2009) Type 2 diabetes mellitus, hypertension, 
dyslipidemia and obesity: a systematic comparison of their impact on cognition. Biochim Biophys Acta 1792:470-481

20. Klein R, Klein BE, Magli YL et al (1986) An alternative method of grading diabetic retinopathy. Ophthalmology 93:1183-1187

21. Ryan CM, Geckle MO (2000) Circumscribed cognitive dysfunction in middle-aged adults with type 2 diabetes. Diabetes Care 23:14861493

22. Schmidt M (1996) Rey auditory verbal learning test: a handbook. Western Psychological Services, Los Angeles

23. Shin MS, Park SY, Park SR, Seol SH, Kwon JS (2006) Clinical and empirical applications of the Rey-Osterrieth Complex Figure Test. Nat Protoc 1:892-899

24. Brucki SM, Rocha MS (2004) Category fluency test: effects of age, gender and education on total scores, clustering and switching in Brazilian Portuguese-speaking subjects. Braz J Med Biol Res 37: 1771-1777

25. Bowie CR, Harvey PD (2006) Administration and interpretation of the Trail Making Test. Nat Protoc 1:2277-2281

26. Samton JB, Ferrando SJ, Sanelli P, Karimi S, Raiteri V, Barnhill JW (2005) The clock drawing test: diagnostic, functional, and neuroimaging correlates in older medically ill adults. J Neuropsychiatry Clin Neurosci 17:533-540

27. Wahlund LO, Barkhof F, Fazekas F et al (2001) A new rating scale for age-related white matter changes applicable to MRI and CT. Stroke 32:1318-1322

28. Song XW, Dong ZY, Long XY et al (2011) REST: a toolkit for resting-state functional magnetic resonance imaging data processing. PLoS One 6, e25031

29. American Diabetes Association (2014) Executive summary: standards of medical care in diabetes - 2014. Diabetes Care 37:S5-S13

30. Uddin LQ, Kelly AM, Biswal BB, Castellanos FX, Milham MP (2009) Functional connectivity of default mode network components: correlation, anticorrelation, and causality. Hum Brain Mapp 30:625-637

31. Jones DT, Machulda MM, Vemuri P et al (2011) Age-related changes in the default mode network are more advanced in Alzheimer disease. Neurology 77:1524-1531

32. Zhu X, Wang X, Xiao J et al (2012) Evidence of a dissociation pattern in resting-state default mode network connectivity in firstepisode, treatment-naive major depression patients. Biol Psychiatry 71:611-617

33. Starck T, Nikkinen J, Rahko J et al (2013) Resting state fMRI reveals a default mode dissociation between retrosplenial and medial prefrontal subnetworks in ASD despite motion scrubbing. Front Hum Neurosci 7:802
34. Jones DT, Vemuri P, Murphy MC et al (2012) Non-stationarity in the "resting brain's" modular architecture. PLoS One 7, e39731

35. Andrews-Hanna JR, Snyder AZ, Vincent JL et al (2007) Disruption of large-scale brain systems in advanced aging. Neuron 56:924-935

36. Machulda MM, Jones DT, Vemuri P et al (2011) Effect of APOE epsilon 4 status on intrinsic network connectivity in cognitively normal elderly subjects. Arch Neurol 68:1131-1136

37. Tregellas JR, Tanabe J, Rojas DC et al (2011) Effects of an alpha 7nicotinic agonist on default network activity in schizophrenia. Biol Psychiatry 69:7-11

38. Leech R, Sharp DJ (2014) The role of the posterior cingulate cortex in cognition and disease. Brain 137:12-32

39. Cui Y, Jiao Y, Chen YC et al (2014) Altered spontaneous brain activity in Type 2 diabetes: a resting-state functional MRI study. Diabetes 63:749-760

40. Hoogenboom WS, Marder TJ, Flores VL et al (2014) Cerebral white matter integrity and resting-state functional connectivity in middle-aged patients with type 2 diabetes. Diabetes 63:728-738

41. Sheline YI, Barch DM, Price JL et al (2009) The default mode network and self-referential processes in depression. Proc Natl Acad Sci U S A 106:1942-1947

42. Musselman DL, Betan E, Larsen H, Phillips LS (2003) Relationship of depression to diabetes types 1 and 2: epidemiology, biology, and treatment. Biol Psychiatry 54:317-329

43. Busing KA, Schonberg SO, Brade J, Wasser K (2013) Impact of blood glucose, diabetes, insulin, and obesity on standardized uptake values in tumors and healthy organs on $18 \mathrm{~F}-\mathrm{FDG}$ PET/CT. Nucl Med Biol 40:206-213

44. Euser SM, Sattar N, Witteman JC et al (2010) A prospective analysis of elevated fasting glucose levels and cognitive function in older people: results from PROSPER and the Rotterdam Study. Diabetes 59:1601-1607

45. Van den Berg E, Kessels R, Kappelle L et al (2006) Type 2 diabetes, cognitive function and dementia: vascular and metabolic determinants. Drugs Today (Barc) 42:741-754

46. Baker LD, Cross DJ, Minoshima S et al (2011) Insulin resistance and Alzheimer-like reductions in regional cerebral glucose metabolism for cognitively normal adults with prediabetes or early type 2 diabetes. Arch Neurol 68:51-57

47. Willette AA, Xu G, Johnson SC et al (2013) Insulin resistance, brain atrophy, and cognitive performance in late middle-aged adults. Diabetes Care 36:443-449

48. Ott A, Stolk R, Van Harskamp F et al (1999) Diabetes mellitus and the risk of dementia The Rotterdam Study. Neurology 53:19371942 